\title{
X-Ray Standing Waves on Surfaces
}

\author{
Héctor D. Abruña \\ Department of Chemistry \\ Baker Laboratory \\ Cornell University \\ Ithaca, New York 14853-1301
}

Table of Contents:

1. Introduction

2. X-ray Standing $\mathrm{W}$. ves Based on Bragg Reflection

3. Total External Reflection X-ray Standing Waves

4. Experimental Aspects

5. Data Analysis

6. Selected Examples

6.1 Bromine adsorption on Si (220)

6.2 Packing density and potential dependent distributional changes of iodine on $\mathrm{Pt}$

6.3 X-ray standing wave study of a Langmuir-Blodget multilayer film

7. Conclusions

8. Acknowledgments

9. References

1. Introduction

This document bas been approved for public selease and sale; it

distribution is unlimited

The $\mathrm{x}$-ray standing wave technique is a sensitive tool for determining the position of atoms within a crystal, adsorbed onto a surface or distributed within the crystal or at the interface. The technique is based on the $x$-ray standing wave field that arises as a result of the interference of coherently related incident and reflected plane waves (Figure 1) and is described by the theory of dynamical diffraction of $x$-rays. [1-3] When two coherently related traveling plane waves having the same wavelength pass through each other their 
superposition results in a standing wave of period $D=\lambda / 2 \sin \theta$; where $\lambda$ is the wavelength of the traveling waves, and $2 \theta$ is the relative angle between them. The generation of a standing wave requires both an incident and a reflected wave and the letter can be generated by either Bragg diffraction or total external reflection.

Conventional XSW are generated using dynamical Bragg reflection [1-3] from perfect single crystals[4-10]. In this case the periodicity of the standing wave field is equivalent to the d-spacing of the (hkl) diffracting planes and, thus, this technique is a precise tool ( $\pm 1 \%$ of the $\mathrm{d}$-spacing)[5] for measuring bond-lengths between adsorbate atoms and surface/bulk lattice positions over the range of 1 to $4 \AA$ (i.e. typical d-spacings for single crystals). In Bragg diffraction XSW measurements, distances are extracted modulo-d (that is relative to the diffraction planes) so that it is inappropriate to employ this method for structural determinations of systems extending over several tens of angstroms such as ionic distributions at charged interfaces. In this case, XSW having a longer perind would be much more appropriate. Such long period XSW can be generated by Bragg diffraction from layered synthetic microstructures (LSM)(vide-infra) which have dspacings ranging from 20 to $200 \AA$ [11-13] or by employing total external reflection from a mirror surface (vide infra) $[14,15]$. The longer periods are due to the fact that at a given wavelength the reflection occurs at smaller angles $(\theta)$ than in the case of Bragg diffraction from single crystals. We will discuss these various techniques and present examples on their applicability.

\section{X-ray Standing Waves Based on Bragg Reflection}

For the case of XSW based on Bragg reflection, the standing wave can extend well beyond the reflecting surface and estimates of this coherence length range to values as large as $1000 \AA$ [12]. The nodal and antinodal planes of the standing wave are parallel to the diffracting planes and as mentioned above, the nodal wavelength corresponds to the dspacing of the diffracting planes. As the angle of incidence is advanced across the strong 
Bragg reflection, the relative phase between the incident and reflected plane waves (at a fixed point in the crystal) changes by $\pi$. Due to this phase change, the antinodal planes of the standing wave field move in the $-\mathrm{H}$ direction normal to the diffraction planes by $1 / 2$ of a $d$-spacing, from a position halfway between the (h,k,l) diffracting planes (low angle side of the Bragg reflection) to a position that coincides with them (high angle side of the Bragg reflection). Thus, the standing wave can be made to sample an adsorbed or distributed overlayer at varying positions above the substrate interface.

For an atomic overlayer which is positioned parallel to the diffracting planes, the nodal and antinodal planes of the standing wave will pass through the atom plane as the angle is advanced. Using an incident $x$-ray beam energy at or beyond the absorption edge of the atoms in the overlayer, the fluorescence emission yield will be modulated in a characteristic fashion as the substrate is rocked in angle. The yield can be expressed as an integral that incorporates a distribution function $\mathrm{f}(\mathrm{z})$ for the atoms in the adlayer:

$$
Y(z, \theta)=\int I(z, \theta) f(z) d z
$$

Figure 2 depicts the angular dependence of the electric field intensity (or fluorescence yield) for an adsorbate layer located at varying positions with respect to the diffracting planes. The phase and amplitude of this modulation (or so-called coherent position and coherent fraction) are a measure of the mean position $\langle z\rangle$ and width $V\langle z\rangle{ }^{2}$, respectively of the distribution $F(z)$ of atoms in the overlayer. The coherent fraction $\left(f_{\mathcal{C}}\right)$ and coherent position $\left(\phi_{c}\right.$; which is equal to $(\langle z>/ d)$ ) are defined as the amplitude and phase, respectively, of the $\mathrm{m}^{\text {th }}$ Fourier component for the distribution of atoms in the adlayer, and are incorporated into the yield equation as:

$$
. \mathrm{Y}(\theta)=\left|\mathrm{E}_{\mathrm{o}}\right|^{2}\left[\left(1+\mathrm{R}+2 \sqrt{\mathrm{R}} \mathrm{f}_{\mathrm{c}} \cos \left(\mathrm{v}-2 \pi \phi_{\mathrm{c}}\right)\right]\right.
$$

For example if all the atoms were at the same $\mathrm{z}$ position (i.e. a distribution described by a delta function) the coherent fraction would be unity. Conversely, if the atoms were 
randomly distributed the coherent fraction would be zero. It should be mentioned that in this case the yield will be given by $(1+R)$ which represents the limiting case of a random distribution. For intermediate cases the coherent fraction would vary from zero to one. For the case of a gaussian distribution, the coherent fraction can be described by $f_{C}=\exp \left(-2 \pi^{2} 0^{2} / d^{2}\right\}$ which is of the same form as the Debye-Waller expression.

Since the $z$ scale of the Bragg diffraction XSW is mod-d, if several coherent positions (in z) are possible for the adsorbate, a single measurement will not be sufficient to unambiguously determine these positions. Thus, XSW measurements with a different period, and thus different Fourier components, (such as higher order Bragg diffracrion measurements or total external reflection measurements) must be performed to allow such an assignment.

Although the characteristic modulo-d length scale of a few angstroms of single crystals is ideal for determining bond lengths between atom layers at single crystal surfaces, it is inappropriate for the structural determination of systems extending over several tens of angstroms (e.g. ionic distributions at charged surfaces).

An alternative to single crystals is the use of synthetic layered microstructures (LSM)[11-13]. For Bragg diffraction purposes, LSMs are depth-periodic structures consisting of alternating layers of high and low electron density materials (such as tungsten and silicon, or platinum and carbon), and are of high enough quality to produce strung Bragg diffraction. They can be prepared with d-spacings from about 20 to $200 \AA$, thus providing information over a much longer length scale. LSM's have been employed in a number of XSW studies. [16-19]

\section{Total External Reflection $\mathrm{X}$-ray Standing Waves}

Since the refractive index of matter for $x$-rays is less than unity $[20,21]$, when $x$ rays are incident on a mirror surface at an angle smaller than the critical angle, the refracted wave cannot penetrate through the reflecting surface. Under this condition of total external 
reflection, all of the incident wave energy goes into the reflected wave and only an exponentially damped evanescent wave penetrates into the medium below the reflecting surface. At the same time, the incident and specularly reflected plane waves interfere to form a standing wave in the more optically dense medium above the mirror surface. The reflectivity and phase of the reflected plane wave are a function of the angle of incidence. Analogous to $\mathrm{XSW}$ generation via Bragg diffraction, there is a change in the relative phase (by $\pi$ ) between the incident and reflected waves. Regarding the phase at $\theta=0$, the reflected plane wave is completely out of phase with respect to the incident plane wave at the mirror surface. Thus, at $\theta=0$ a node is at the mirror surface and the first antinode is at infinity since $D=\infty$. As the angle of incidence is increased, the first antinode moves inward, in a direction normal to the surface, until at the critical angle it coincides with the mirror surface. The trailing antinodes follow behind with a periodic spacing given by:

$$
D=\lambda /(2 \sin \theta)
$$

In this context one can also define a critical period $D_{\mathcal{C}}$ which is that when $\theta=\theta_{\mathcal{C}}$ where $\theta_{\mathcal{C}}$ is the critical angle. It should be mentioned that for $1 \AA$ wavelength $x$-rays values of $\theta_{c}$ range from $1-10 \mathrm{mrad}$ so that the critical period ranges from 80 to $300 \AA$.

\section{Experimental Aspects}

$\mathrm{X}$-ray standing wave measurements are, in general, experimentally very demanding. An XSW experiment typically consists of monitoring some signal proportional to the standing wave electric field intensity as the angle of incidence is scanned through the total external reflection or across a Bragg reflection. The more commonly employed schemes are electron yield and $\mathrm{x}$-ray fluorescence yield. In the discussion, fluorescence detection will be assumed. A typical experimental set up consists of 1) a collimated beam whose intensity $I_{O}$ is typically monitored with an ionization chamber, 2) a sample stage, 3)a reflected beam monitor $I_{R}$ and 4) a fluorescence detector at $90^{\circ}$ relative to 
the incident $\mathrm{x}$-ray beam. In the case of XSW measurements on single crystals, particular attention needs to be paid to the monochromator design since one needs to be in an nondispersive condition and high collimation is required. Because of this last point, asymmetric crystals are typically employed in the monochromator. Materlik et. at. have recently reviewed many of these experimental details.[22]

At each angular position, a complete $\mathbf{x}$-ray fluorescence spectrum is collected and later analyzed so as to accurately remove background and other undesirable contributions to the signal.

\section{Data Analysis}

Analysis of x-ray standing wave data is based on a fit of the data (reflectivity and fluorescence yield) to those predicted from theory. However, the data must first be treated to extract yields corrected for background and other contributions. In general, the fluorescence yield is recorded as a function of angle of incidence. An energy dispersed spectrum is collected at each angular position. The emission line of interest is then fitted to an assumed functional form (usually a combination of Gaussian functions) and the background is subtracted using polynomial function. From this one obtains the fluorescence yield as a function of the angle of the incident radiation.

The electric field intensity at a given point above the reflecting surface must either be calculated form dynamical diffraction theory [1-3] or from an optical theory approach. The latter approach is generally based on a formalism in which the medium is divided into parallel slabs [23]. The continuity of the tangential components of the electric and magnetic fields at each of the resulting interfaces is the essential requirement invoked to obtain a recursion relation (containing Fresnel coefficients) describing the E-field amplitudes at each interface of the incident, reflected and refracted waves. Such a treatment is applicable to the total external reflection condition as well as to Bragg diffraction. 
The layered medium approach is particularly well suited for analysis of standing waves in multilayered structures [11-13,24]. The recursion relation employed generally has the following form:

$$
R_{j, j+1}=a_{j}^{2}\left[\frac{R_{j+1, j+2}+F_{j, j+1}}{1+R_{j+1, j+2} F_{j, j+1}}\right]=\frac{E_{j}^{R}(o)}{E_{j}(o)}
$$

where

$$
\begin{aligned}
& R_{j, j+1}=\text { ratio of reflected E-field amplitude to incident } \\
& \text { E-field amplitude for } j^{\text {th }} \text { layer } \\
& R_{j+1, j+2}=\text { ratio of reflected wave amplitude to incident } \\
& \text { wave amplinde for } j+1^{\text {th }} \text { layer } \\
& F_{j, j+1}=\text { Fresnel coefficient for } j^{\text {th }} \text { layer } \\
& \text { aj = complex amplitude factor at the } j, j+1 \text { interface. }
\end{aligned}
$$

The reflectivity at a given interface is the squared modulus of $R_{j, j+1}$. The total reflectivity at a given angle of a structure consisting of $n$ layers is obtained by applying the recursion relation n-1 times from the substrate (or from the extinction length) to the topmost layer.

The description in the total external reflection region becomes more complicated since absorption and refraction effects in the region above the reflecting surface can no longer be ignored as is typically done for Bragg diffraction from single crystals.

\section{Selected Examples}

\subsection{Bromine adsorption on Si (220)}

This early study by Golovchenko and co-workers [4] is representative of studies on single crystal surfaces. It is also of note because the sample ( $\mathrm{Si}(220)$ ) was in contact with a liquid (methanol); establishing the feasibility of carrying out in-situ studies on solid/liquid interfaces. The sample consisted of a Si(220) single crystal that was Syton polished and etched with HF and subsequently brought into contact with a solution of bromine in methanol. After rinsing with clean methanol (6x) the sample was covered with a 0.5 mil 
thick film of mylar which trapped a thin film (ca $5 \mu \mathrm{m}$ ) of methanol. The sample was placed in a holder that continually replaced methanol by capillary action. This assembly was then mounted on a high precision stage where the XSW experiments were carried out using $\mathrm{MoK}_{\alpha}$ radiation. The reflectivity across the (220) reflection as well as the $\mathrm{BrK}_{\alpha}$ fluorescence were monitored as a function of angle. The results are presented in Figure 3 where both the reflectivity profile $(A)$ and fluorescence yield $(C)$ are presented along with fits and theoretical calculations (B,D). Figure 3A (o) presents the experimentally measured reflectivity profile along with the best fit. The profile has the characteristic shape (DarwinPrins) anticipated for a nearly perfect crystal. The experimental $\mathrm{BrK} \alpha$ fluorescence yield $(\bullet)$ and the fit are presented in curve $3 \mathrm{C}$. From the fit to the fluorescence yield they determined that the bromine ad-layer was located $1.73 \pm 0.07 \AA$ above the crystal surface and that the coherent fraction was $30 \%$. Also shown in the figure are the anticipated yields for a completely incoherent ad-layer (B) as well as for a layer at $1.73 \AA$ with a $100 \%$ coherent fraction (D). As mentioned previously for an incoherent layer the yield will be given by $(1+R)$ as seen in the figure. For a completely coherent layer, we observe a very large modulation. The experimental results have a clear resemblance to the latter, but the amplitude of the modulation is clearly less.

\subsection{Packing density and potential dependent distributional changes of iodine on Pt:}

The $\mathrm{Pt}(111) / \mathrm{I}$ system possesses a rich potential-dependent coverage isotherm, which has been characterized by Auger spectroscopy [25-27] for the emersed case, and by $x$-ray absorption spectroscopy in-situ [28]. Its features are explained in terms of potential dependent structural and distributional changes. The aim in this work was to study, insitu, via the XSW method using a Pt/C Layered Synthetic Microstructure (LSM), the potential dependence of structural and distributional changes of iodine species, and to relate features of the aforementioned isotherm to observations from this measurement [18]. 
In the interpretation of the data, we chose a relatively simple model (Figure 4A) consisting of three basic components: (1) an ad-layer (step) of specifically adsorbed (i.e.. in contact with the electrode surface) iodine atoms, (2) an exponential tail of iodide extending out into solution with a characteristic decay length $k$, (the diffuse layer), and (3) a second layer (step) of width equal to the thickness of the solution layer, depicting bulk iodide. The effect of including a diffuse layer, along with the adsorbed layer in the model corresponds to a superposition of a random-like component to the coherent XSW yield from the ad-layer (provided that the thickness $t_{\text {ad }}$ is narrow with respect to the substrate's d-spacing), in a ratio proportional to the population in each layer with the number of atoms in the diffuse layer being controlled by the decay length $k$ and the initial concentration $\mathrm{N}_{\text {diff }}$

The angular dependence of the IL fluorescence yield for each of the potentials studied is shown in Figure 5. Changes in the phase (angular position) and amplitude of the standing wave signal are indicative of distributional changes in the direction normal to the substrate surface. We also observe different vaiues of the off-Bragg fluorescence yield at different applied potentials corresponding to changes in the total amount of $I^{-} I$ species sampled by the incident and reflected beams. In addition, the potential dependent changes in the background slope, peak position, and modulation amplitude provide further insights about the distribution of species.

Based on an analysis of the standing wave and off-Bragg yield measurements, we note a marked accumulation of iodide in the diffuse layer, weakly associated with the adsorbed iodine, when the $\mathrm{Pt}$ surface is not saturated by iodine adatoms $(-0.1 \mathrm{~V})$. This striking association of iodide with the iodine ad-lattice is perhaps driven by the hydrophilic character of the unsaturated Pt surface. In addition, the increase in the adsorbed iodine packing density to saturation coverage is accompanied by an abrupt decrease in the concentration of this accumulated iodide. Thus, the potential dependent structural transformation in the iodine ad-lattice might be viewed as a phase transition, in which 
iodide anions in the liquid-like arrangement of the diffuse layer are incorporated into the crystalline-like structure of the iodine ad-lattice. This results in a saturated, possibly hydrophobic Pt surface and in the concomitant decrease in the concentration of iodide in the diffuse layer associated with the adsorbed iodine. These changes are depicied graphically in Figure 4B.

\subsection{X-ray standing wave study of a Langmuir-Blodgett multilayer film}

As mentioned previously the total external reflection condition occurs between $\theta=0$ and $\theta=\theta_{c}$ (where $\theta_{c}$ is of the order of a few milliradians). Over this angular range the period of the generated XSW varies between infinity and several nanometers in length. This characteristically long and variable period distinguishes the total external reflection XSW from the Bragg diffraction XSW where the period is essentially dictated by the dspacing of the diffracting planes. The similarity between the two cases is that a $\pi$ radian phase shift occurs, in this case as the incident angle is advanced through the total reflection condition. As in the previous cases, the signal (e.g. fluorescence) arising from a marker atom can be used to ascertain the angular dependence of the XSW field. However, it should be noted that in this case the period of the standing wave is continuously changing. Also since large periods are involved, the marker atom must be placed at a mean distance $(<z>)$ that is larger than the critical period for the mirror material and the distribution $\left(\sqrt{ }<z^{2}>\right)$ of the marker atom must be narrow relative to $(\langle z>)$. If these conditions are satisfied, then there will be $\left(<z>/ D_{c}+I / 2\right)$ modulations in the fluorescence yield of the marker atom between $\theta=0$ and the critical angle.

The use of XSW based on mirror reflection was first demonstrated by Bedzyk and co- workers who measured the fluorescence yield for a $\mathrm{Zn}$ atom layer embedded in the top arachidate bilayer of a Langmuir-Blodgett (LB) multilayer film which was deposited on the surface of a gold mirror [14]. As shown in Figure 6, three full oscillations in the $\mathrm{Zn}$ fluorescence yield occur before reaching the critical angle of gold (arrow in figure), 
indicating that the $\mathrm{Zn}$ layer was located at $\angle \mathrm{Z}\rangle=2.5 \mathrm{D}_{\mathrm{c}}=200 \AA$. (The critical angle for gold at the incident energy of $9.8 \mathrm{keV}$ is about $7.8 \mathrm{mrad}$ so that the critical period $\mathrm{D}_{\mathrm{c}}$ is about $80 \AA$. The va!. of $<z>$ was more precisely determined to be $218 \AA$ from a $\chi^{2}$ fit of the data to theoretical yields based on a layered model. This value was also in very good agreement with calculations based on the dimensions of the deposited layers. Since the standing wave samples the selected atom distribution with a variable period $D$, one is, in effect. measuring the Fourier transform of that distribution over a continuous range in $Q=$ 1/D (i.e. a scan in Fourier space). With a variable period ranging from 10's of angstroms to 100 's of angstroms, this $\mathrm{x}$-ray standing wave technique is ideally suited to measure surface and interface layered structures which have natural length scales in the 10 to $1000 \AA$ regime.

\section{Conclusions and Future Directions:}

The use of $x$-ray standing waves is yielding valuable insights on interfacial structure and composition. Future applications of this novel technique to the study of interfaces will yield extremely important and fundamental information that will affect not only our understanding and control of interfacial reactivity, but the field of surface chemistry as a whole.

\section{Acknowledgments:}

Our work has been supported by the Office of Naval Research, the National Science Foundation, the Army Research Office and the Materials Science Center at Cornell University. 
9. References:

1 von Laue, M.; Roentgenstrahlinterenzen (Akademische Verlargsgesellshaft. Frankfurt. 1960).

2. Batterman, B.W.; Cole, H.; Rev. Mod. Phys., 1964, 36, 681.

3. Batterman, B.W.; Phys. Rev.. 1964, 133, A759.

4. Cowan, P.L.; Golovchenko, J.A.; Robbins, M.F.; Phys. Rev. Lett., 1980, $44,1680$.

5. Bedzyk, M.J.; Materlik, G.; Phys. Rev. B, 1985, 31, 4110.

6. Zegenhagen, J.; Huang, K.-G.; Gibson, W.M.; Hunt, B.D.; Schowalter, L.J.; Phys. Rev. B, 1989, 39, 10254.

7. Woodruff, D.P.; Seymour, D.L.; McConville, C.F.; Riley, C.E.; Crapper, M.D.; Prince, N.P.; Jones, R.G.; Phys. Rev. Lett., 1984, 58, 1480.

8. Woodruff, D.P.; Seymour, D.L.; McConville, C.F.; Riley, C.E.; Crapper, M.D.; Prince, N.P.; Jones, R.G.; Surface Science, 1988, 195, 237.

9. Materlik, G.; Zegenhagen, J.; Uelhoff, W.; Phys. Rev. B, 1985, 32, 5502.

10. Materlik, G.; Schmah, M.; Zegenhagen, J.; Uelhoff, W.; Ber. Bunsenges. Phys. Chem., 1987, 91, 292.

11. Underwood, J.H., Barbee, T.W.; in AIP Conf. Proc., 75, 170; D.T. Atwood, B.L. Henke, eds., AIP, New York, 1981.

12. Barbee, T.W.; Underwood, J.H.; Optics Comm., 1983, 48, 161.

13. Barbee, T.W.; Warburton, W.K.; Mater. Lett., 1984, 3, 17.

14. Bedzyk, M.J.; Bommarito, G.M.; Schildkraut, J.S.; Phys. Rev. Lett., 1989, 62, 1376.

15. Bedzyk, M.J.; Bommarito, G.M.; Caffrey, M.; Penner, T.L.; Science, 1990, 248 , 52.

16. Bedzyk, M.J.; Bilderback, D.; White, J.H.; Abruña, H.D.; Bommariio, G.M.; $J$. Phys. Chem., 1986, 20, 4926. 
17. Bedzyk, M.J.; Bilderback, D.H.; Bommarito, G.M.; Caffrey, M.; Schildkraut, J.S.; Science, 1988, 241, 1788.

18. Bommarito, G.M.; White, J.H.; Abruña, H.D.; J. Phys. Chem., 1990, 24, 8280.

19. Abruña, H.D.; Bommarito, G.M.; Acevedo, D.; Science, 1990, 250, 69.

20. James, R.W.; The Optical Principles of the Diffraction of X-ravs," Oxbow Press, Woodbridge, Connecticut, 1982.

21. Bilderback, D.H.; SPIE Proc., 1982, $115,90$.

22. Zegenhagen, J.; Materlik, G.; Uelhoff, W.; Inl. X-ray Sci. \& Tech., 1990, 2, 214.

23 Parratt, L.G.; Phys. Rev., 1954, 25, 359.

24 Bommarito, G.M.; M.S. Thesis, Cornell University, 1987.

25. Felter, T.E.; Hubbard, A.T.; J. Electroanal. Chem., 1979, 100, 473.

7.6. Garwood, G.A.; Hubbard. A.T.; Surf. Sci.. 1980, $22,617$.

27. Salaita, G.N.; Baltruschat, H.; Hubbard, A.T.; J. Electroanal. Chem., 1987, 222, 305.

28. White, J.H.; Ahruña, H.D.; J. Phys. Chem., 1988, $22,7131$. 
Figure Legends

Figure 1. Illustration of the $\mathrm{x}$-ray standing wave generated by the interference of coherently related incident and reflected plane waves above a reflecting surface.

Figure 2. Angular dependence of the reflectivity and the electric filed intensity at various positions with respect to the diffraction planes (adapted from ref. 19)

Figure 3. Normalized $\mathbf{x}$-ray yield from fluorescence and reflected beam detectors for $\mathrm{Br}$ adsorbed on Si(220): (o) Bragg reflectivity; $(\bullet$ ) bromine fluorescence.

Curves:
A. Bragg reflectivity theory
B. standing wave theory with zero coherent fraction
C. standing wave theory with $30 \%$ coherent fraction at a position $1.72 \AA$ above the surface
D. standing wave theory with $100 \%$ coherent fraction at a position $1.72 \AA$ above the surface

(figure adapted from ref.4)

Figure 4 A. Schematic of the model used to describe the iodide distribution formed at a platinum electrode surface.

B. Illustration of the changes in the iodine/iodide distribution at a platinum electrode surface as a function of applied potential.

(figure adapted from ref. 18)

Figure $j$ The angular dependence of the experimental $I_{L}$ fluorescence for the various applied potentials studied and experimental reflectivity at $6.0 \mathrm{keV}$.

(figure adapted from ref. 18)

Figure 6 The angular dependence of the $\mathrm{Zn} \mathrm{K}_{\alpha}$ fluorescence yield from a LangmuirBlodgett film consisting of zine arachidate, $\omega$-tricosanoic acid and cadmium arachidate. Arrow indicates the critical angle for the gold surface.

(figure adapted from ref.14) 


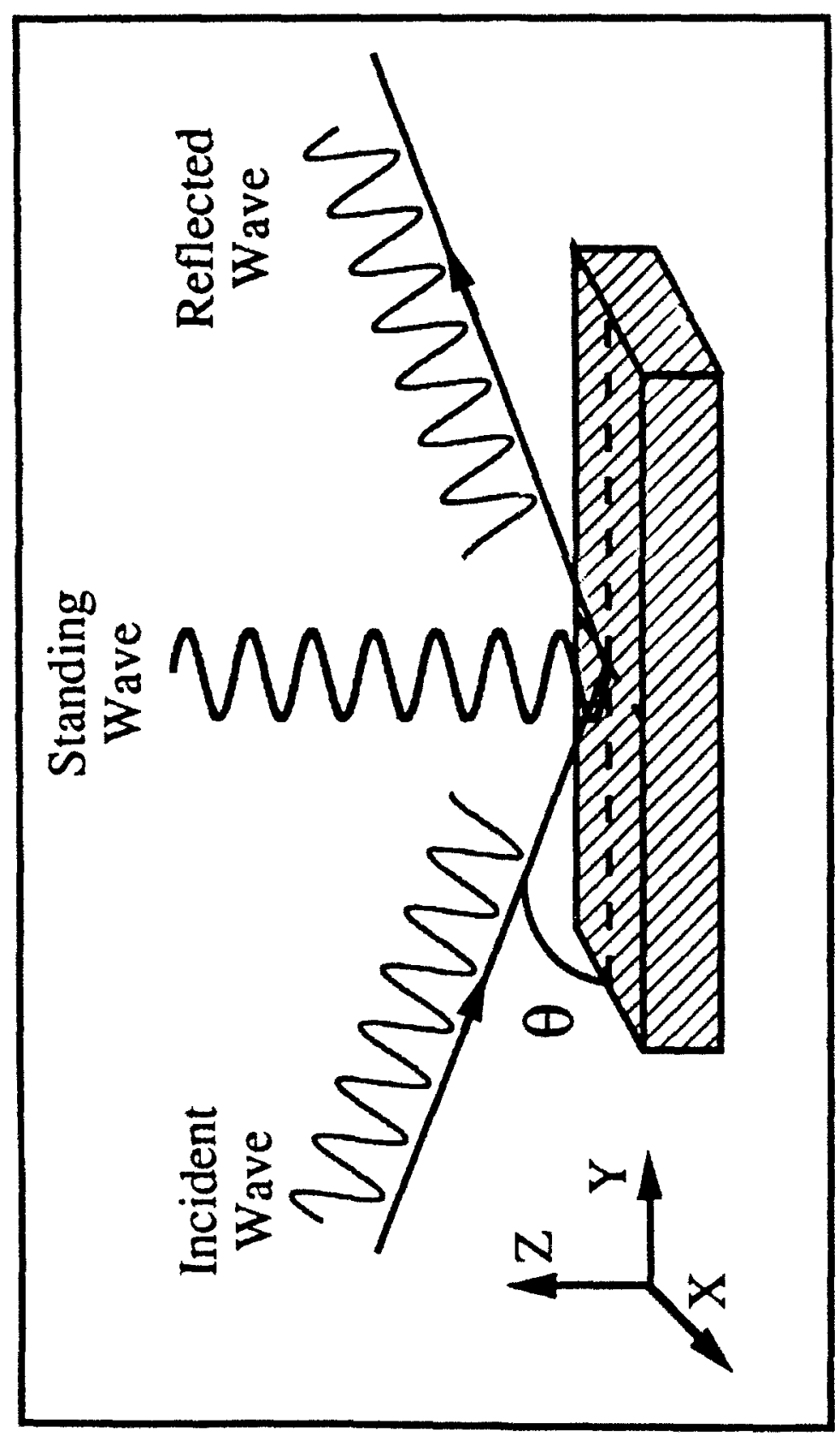

$\underset{10}{0}$ 


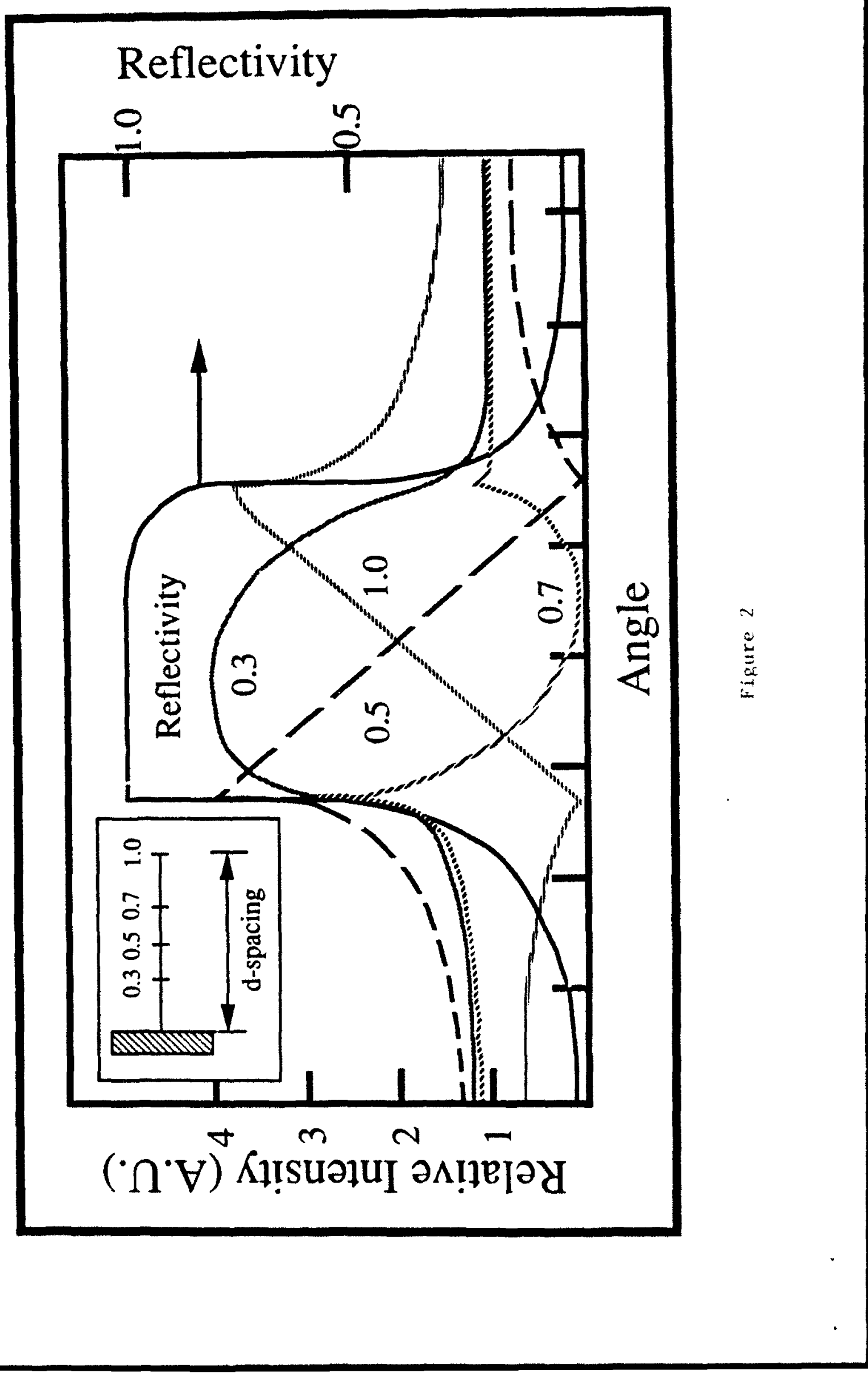




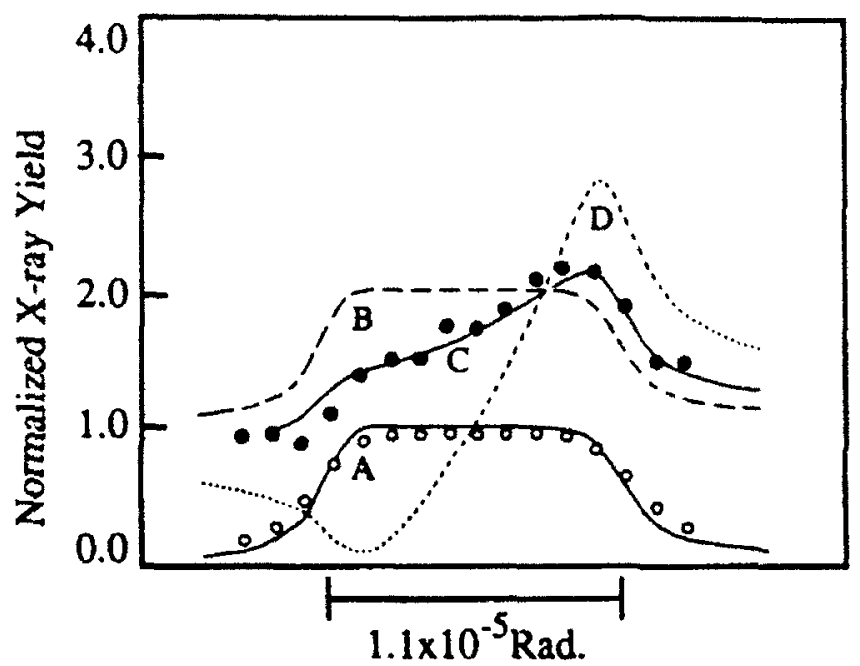

Figure 3 


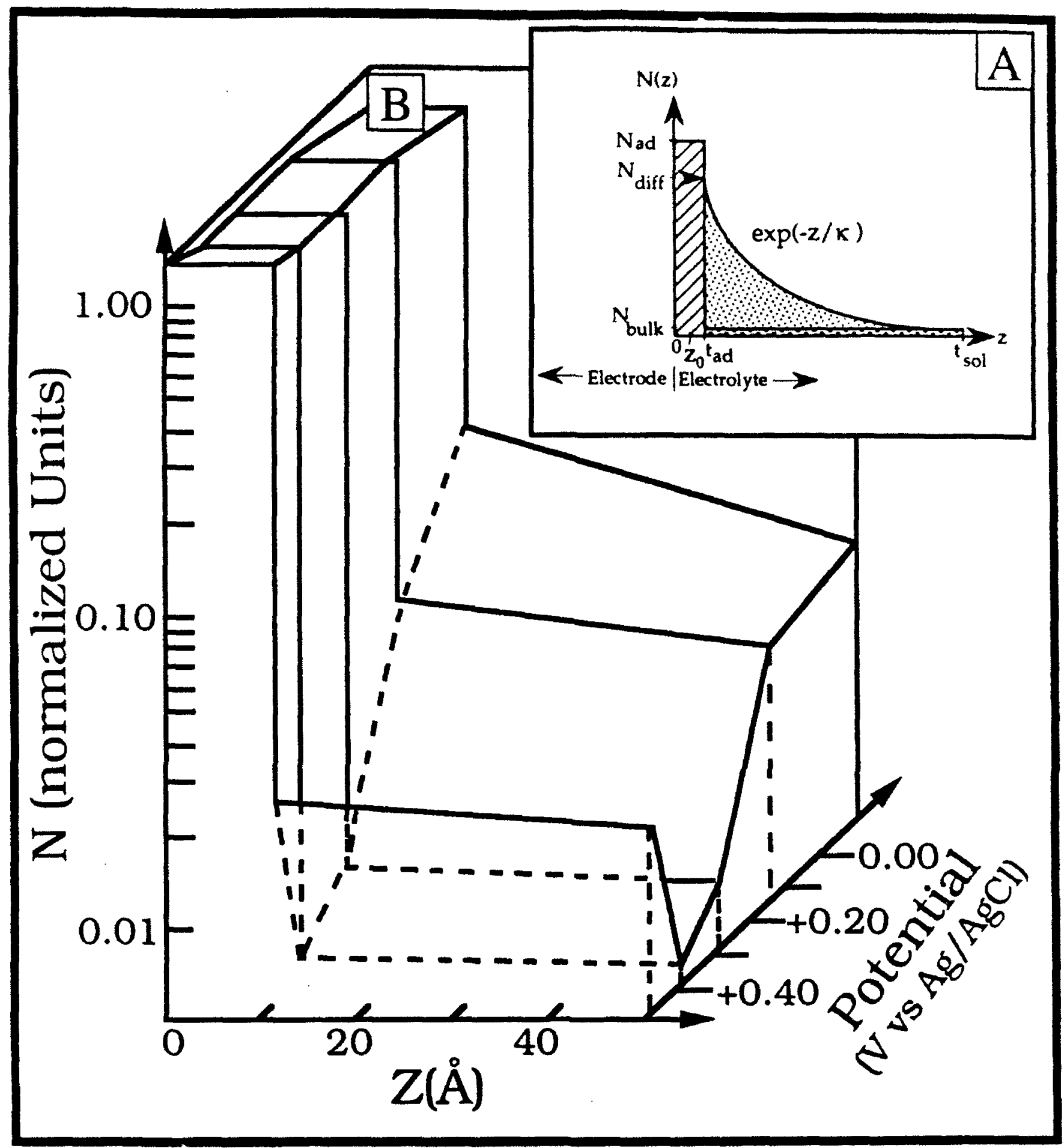

Figure 4 


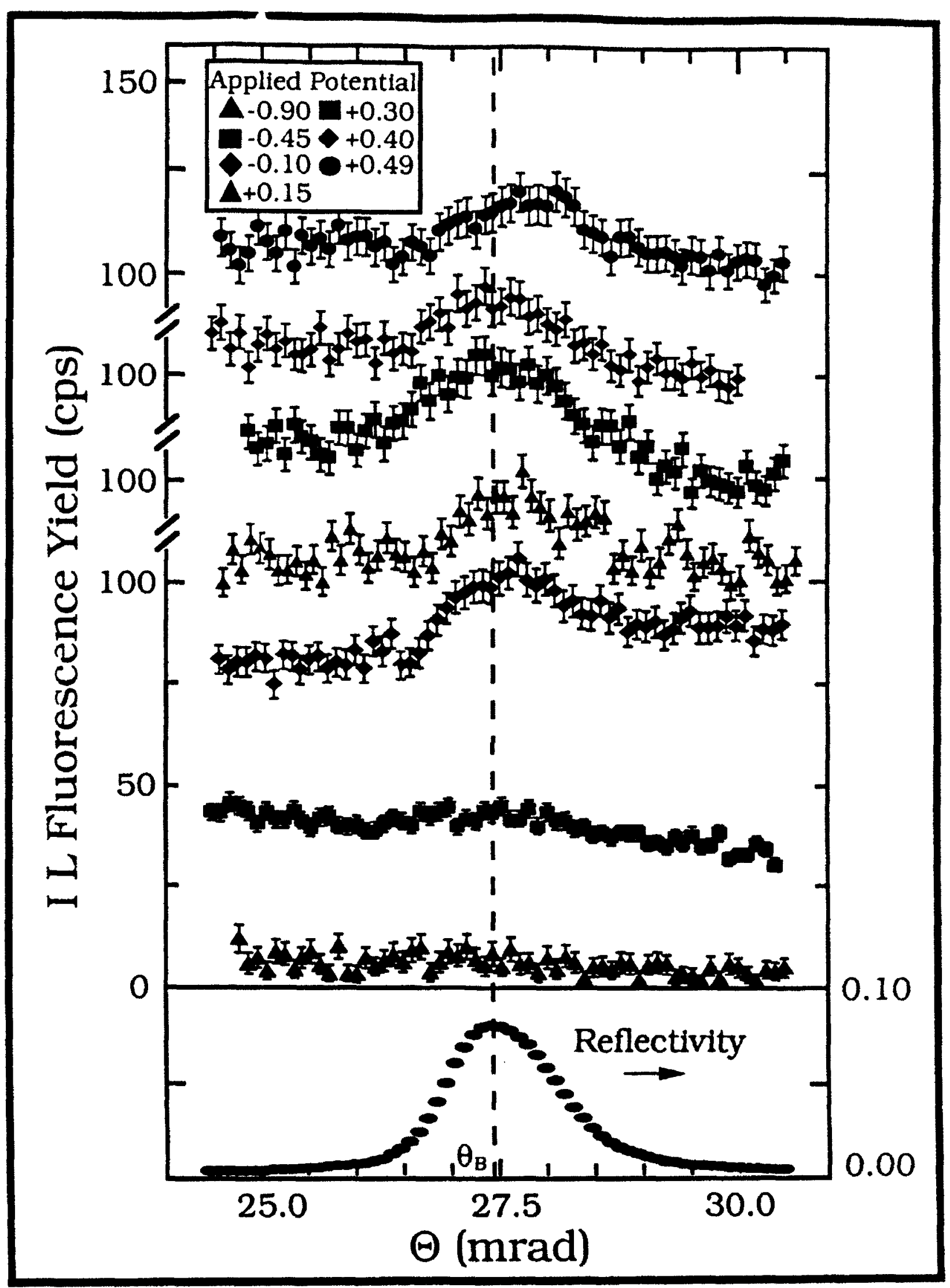

Figure 5 


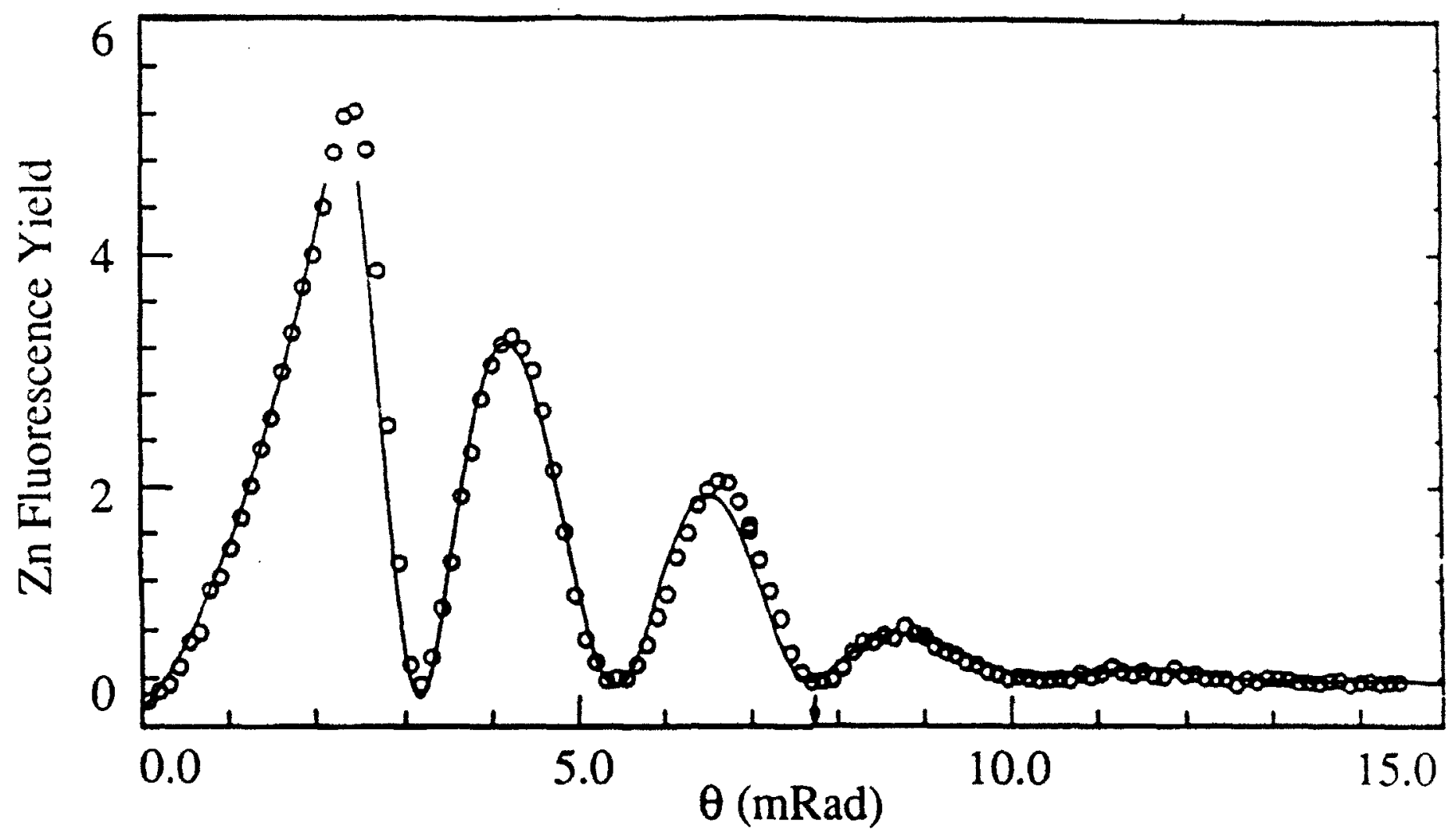

Figure 6 